\title{
Tecnura
}

\section{Sistemas piezoeléctricos en el tren urbano de Guadalajara, México: entropía y negentropía}

\section{Piezoelectric systems in the urban train of guadalajara, Mexico: entropy and negentropy}

\author{
Arturo Arizaga Barragán¹, Mario Guadalupe González Pérez², Yefer Asprilla Lara
}

Fecha de recepción: 12 de febrero de 2019

Fecha de aceptación: 15 de mayo de 2019

Como citar: Arizaga B., A., González P., M.G.y Asprilla L., Y. (2019). Sistemas piezoeléctricos en el tren urbano de Guadalajara, México: entropía y negentropía. Tecnura, 23(61), 13-22. doi: https://doi.org/ 10.14483/22487638.14870

\section{Resumen}

Contexto: La homeostasis del tren urbano de Guadalajara, México, experimenta entropía relativa a la aglomeración de usuarios en las denominadas horas pico. Esto conlleva un mayor consumo de energía eléctrica en los desplazamientos cotidianos; no obstante, el peso proveniente de los usuarios puede ser utilizado como negentropía termodinámica para reducir sus niveles de entropía. En este sentido, el objetivo de esta investigación determina la viabilidad de utilizar la piezoelectricidad en la obtención y consumo de energía eléctrica para la operatividad del sistema.

Método: La investigación se fundamenta en datos proprocionados por el Sistema de Tren Eléctrico Urbano (Siteur) y la Comisión Federal de Electricidad (CFE). Complementariamente, esta información se triangula con datos realizados en ejercicios de campo y en función de la oferta y demanda energética; se calculan el peso aproximado de los usuarios y la energía que puede producir el sistema de generación piezoeléctrica por persona. Finalmente, es utilizado el modelo entropía-homeostasis-negentropía (EHN), para determinar el escenario de la piezoelectricidad en el consumo energético del tren eléctrico urbano de la metrópoli de Guadalajara.

Resultados: La utilización de piezoeléctricidad puede lograr eficiencias significativas para la optimización energética de sistemas de movilidad urbana; inclusive de hasta un 89,7 \% como en el ejemplo de la línea 2 del Siteur en Guadalajara. Esto por las condiciones homeostáticas propias de este sistema, relativas a la afluencia promedio diaria en horas pico; cuando se llega a los 83.059 usuarios. Por tanto, es posible generar negentropía termodinámica, a través de la energía eléctrica proveniente de los sistemas piezoeléctricos y el peso de los usuarios.

Conclusiones: La energía autoproducida por piezoelectricidad en el sistema, mediante el aprovechamiento del peso de los usuarios puede ser almacenada para alimentar la líneas de la red del tren eléctrico urbano. De esta manera, se optimiza el consumo energético en los desplazamientos cotidianos, con ahorros económicos para la empresa y

1 Ingeniero en Energía, División de Ingenierías e Innovación Tecnológica. Centro Universitario de Tonalá, Universidad de Guadalajara. Guadalajara, México. Contacto: arturo.arizaga@alumno.udg.mx ORCID: https://orcid.org/0000-0002-3109-4375

2 Ingeniero civil, maestro en Ingeniería, doctor en Ciudad, Territorio y Sustentabilidad. Profesor titular, Centro Universitario de Tonalá, Universidad de Guadalajara. Guadalajara, México. Contacto: mario.gonzalez@academico.udg.mx ORCID: https://orcid.org/0000-0002-5457-5948

3 Ingeniero civil, especialista en Infraestructura Vial, magíster en Ingeniería Civil, candidato a doctor en Movilidad Urbana, Transporte y Territorio. Docente de la Universidad Distrital Francisco José de Caldas. Bogotá, Colombia. Contacto: yasprillal@udistrital.edu.co ORCID: https://orcid.org/0000-0001-6569-7441 
como negentropía en la reducción de la energía no utilizada para producir trabajo (entropía).

Palabras clave: entropía, negentropía, piezoelectricidad, consumo energético, tren eléctrico

\begin{abstract}
Context: The homeostasis of the Urban Train of Guadalajara (Mexico) experiences entropy relative to the conglomeration of users in the so-called rush hours. This leads to greater consumption of electrical energy in daily commutes; however, the weight coming from users can be used as thermodynamic negentropy to reduce their entropy levels. Therefore, the objective of this research is to determine the feasibility of using piezoelectricity in obtaining and consuming electrical energy for the operation of the system.

Method: The research is based on the data provided by the Urban Electric Train System (SITEUR, in Spanish) and the Federal Electricity Commission (CFE). This information is triangulated along with data obtained in field exercises, and based on energy the supply and demand; then, the approximate weight of the users and the energy that the piezoelectric generation system can produce per person are calculated.
\end{abstract}

Finally, the Entropy-Homeostasis-Negentropy (EHN) model is used to determine the homeostasis of piezoelectricity in the energy consumption of the urban electric train in the metropolis of Guadalajara.

Results: The use of piezoelectricity can significantly improve efficiency and achieve energy optimization of urban mobility systems up to $89,7 \%$; for example, the case of Line 2 of the SITEUR in Guadalajara. This improvement in efficiency is possible due to the homeostatic conditions of the system and the average influx during rush hours, when it reaches 83,059 users. Therefore, it is possible to generate thermodynamic negentropy through the electrical energy coming from the piezoelectric systems and the weight of the users.

Conclusions: The energy produced by piezoelectricity that makes use of the users' weight can be stored to power the lines of the urban electric train network. In this way, the efficiency of the energy consumption in daily commutes is improved, saving the company money and energy (through the reduced amount use of external energy to produce work).

Keywords: entropy, negentropy, piezoelectricity, energy consumption, electric train.

\section{INTRODUCCIÓN}

En México, tres ciudades metropolitanas integran sistemas de transporte eléctrico a su movilidad urbana: Ciudad de México, Guadalajara y Monterrey. En cuanto a líneas de operación y movilidad de usuarios, el más grande es el Sistema de Transporte Colectivo de la Ciudad de México (Metro). Tan solo en 2006, el denominado Metro ocupó el tercer lugar a nivel mundial al transportar a una media de 3,9 millones de pasajeros al día; incluso, superando los metros de Nueva York, Moscú y Tokio. Asimismo, ese año obtuvo el quinto lugar en el mundo por la extensión de su red (Sistema de Transporte Colectivo, 2007).

En este contexto, en años recientes la metrópoli de Guadalajara ha evidenciado "insuficiencia y deficiencia de conectividad, accesibilidad y medios adecuados de transporte" (González, 2016). Además, la urbe experimenta un incremento poblacional anual y según el Instituto de Información Estadística y Geografía del Estado de Jalisco (IIEG) ha llegado a los cinco millones de habitantes (IIEG, 2017), con más de dos millones de unidades vehiculares operando (De Quevedo, Asprilla y González, 2017, p. 138). Este escenario afecta los procesos de movilidad urbana supeditada a la automovilidad, debido a la afluencia de personas que intentan desplazarse diariamente desde sus orígenes a sus destinos, y provocan conflictos viales durante las denominadas horas pico. De esta manera, diferentes ciudades de carácter metropolitano supeditadas a la automovilidad han comprometido la sustentabilidad de sus recursos y han 
ocasionado graves problemas de índole económica, social y ambiental.

El estado actual del sistema de movilidad urbana en la metrópoli de Guadalajara (homeostasis) se encuentra en desequilibrio dinámico, debido a que la entropía (desestabilización sistémica) generada por la industria inmobiliaria y su cadena de distribución, la industria automotriz y de autopartes, los proveedores del servicio de transporte, las empresas de venta de combustibles y lubricantes, y en general los intereses de los grupos que ostentan temporalmente la representación social son mayores a la capacidad de carga del sistema, y por tanto, rebasan al conjunto de fuerzas tendientes a generar neguentropía (estabilización sistémica). (García, González y Asprilla, 2018, p. 63)

En el ámbito económico se ha identificado una continua pavimentación y artificialización del suelo, grandes construcciones de concreto y asfalto, que ocasionan costos indirectos para el mantenimiento de la señalización, semaforización o conservación de la cubierta. En materia social se ha favorecido la accidentalidad y tiempos muertos ocasionados por la congestión del tráfico, daño físico o mental causado por la emisión de gases, ruido y estrés (González, 2018, p. 72). Asimismo, en el aspecto ambiental se han alterado muchas veces irreversiblemente la topografía del terreno natural, por medio de vibraciones inducidas por el tráfico pesado; eliminación de la cubierta vegetal -que incrementa la velocidad de las escorrentías superficiales y la erosión del suelo-; contaminación de los sistemas de drenaje a causa de los derivados del petróleo de la combustión interna de los vehículos; contaminación de cuerpos de agua, superficiales y freáticos por desechos sólidos urbanos adyacentes a las vías; entre otras fuentes contaminantes.

En un entorno con panoramas de gran oferta de energía y un crecimiento sostenido de la demanda, las políticas energéticas de los países desarrollados deben Ilevar un equilibrio necesario entre los objetivos de aumento de competitividad medioambiental y seguridad en el abastecimiento. (Faure, 2004)

Los sistemas urbanos no dependientes de la automovilidad han venido a reducir la entropía; es decir, a producir negentropía termodinámica. Tal es el caso del denominado Sistema de Tren Eléctrico Urbano, el cual está constituido por dos líneas: a) línea 1 en sentido norte-sur, que transita desde la estación Periférico-Norte hasta la estación Periférico-Sur, contiene 19 estaciones en 15,5 $\mathrm{km}$ de longitud, y b) línea 2 en sentido occidente-oriente, parte desde la estación Juarez hasta la estación Tetlán, y cuenta con 10 estaciones en 8,5 $\mathrm{km}$ de longitud. Es uno de los transportes públicos mayormente utilizados por la población en la metrópoli; a tal grado, que en 2010 prestaba servicio alrededor de 240.000 usuarios diariamente (Mapa Metro, 2010), y para 2016, a más de 245,000 (ISA Corporativo, 2016).

El servicio del sistema de tren eléctrico de Guadalajara opera los 365 días del año, de lunes a domingos, con un horario de 5:00 horas a 23:00 horas. El recorrido que hacen los trenes en la línea 2 es de 36 minutos (ida y vuelta). Dentro del sistema de datos de Siteur se presenta información sobre la frecuencia de paso; es decir, en horas pico, la línea 2 mantiene una frecuencia de 3,35 minutos y en horas valle, de 6,00 a 7,15 minutos. En este sentido, se realizaron una serie de observaciones y mediciones cuantitativas el día martes 5 de diciembre de 2017; con el objetivo de comprobar la frecuencia de paso de los trenes en la hora pico, de las 18:40 hasta las 20:20 horas, considerando esta última hora, porque fue cuando se observó una considerable disminución la cantidad de usuarios que pretendían abordar el tren dentro de la estación Juárez en dirección a la estación Tetlán de la línea 2 de Siteur. Se decidió tomar como punto de partida la estación Juárez, debido que aquí es donde hacen intersección las dos líneas de tren ligero, por lo que cuenta con un gran flujo de usuarios dentro de la estación por los transbordos 
que estos hacen, y además, se encuentra ubicada en el centro de la ciudad de Guadalajara.

Con base en lo anterior, y considerando la segunda ley de Newton, se tiene que a mayor peso (masa), mayor será el gasto energético al momento de moverse el tren; este consume más energía eléctrica cuando se encuentra lleno de usuarios. No obstante, las líneas 1 y 2 del Siteur cuentan con un sistema de generación de energía eléctrica, a través de los vagones al momento del frenado; es decir, esta energía producida es inyectada nuevamente al sistema para ser aprovechada por el siguiente tren (Siteur, 2014). En suma, la utilización de esta tecnología ayuda a hacer eficiente el desplazamiento del sistema y la movilidad de los usuarios; sin embargo, ¿cómo lograr una mayor eficiencia?, o ¿qué factores deben ser considerados para lograr una mayor eficiencia del sistema?

El planteamiento hipotético se centra en "la utilización de la piezoelectricidad como ecotecnología capaz de generar ahorros económicos y energéticos". En este sentido, en función de la oferta y demanda energética en la línea 2 del tren ligero en las horas pico, se obtuvo el peso de los usuarios y la energía por persona que el sistema piezoeléctrico puede producir. Posteriormente, la información documental y los datos obtenidos en campo son trasladados al modelo entropía-homeotasis-negentropía (EHN), para establecer las condiciones homeostáticas del consumo energético del tren eléctrico en la metrópoli. Este modelo retoma los postulados de la segunda ley de la termodinámica y las consideraciones fundacionales de la teoría de sistemas, y logra, como lo muestra la figura 1, una conceptualización que clasifica y califica cualitativamente el impacto antrópico. Los conceptos entropía, homeostasis y negentropía están constituidos por tres subfases cada uno: entropía subcrítica, entropía crítica, entropía hipercrítica, homeostasis reversible, homeostasis cuasirreversible, homeostasis irreversible, negentropía inexistente, negentropía ocasional y negentropía definitiva.

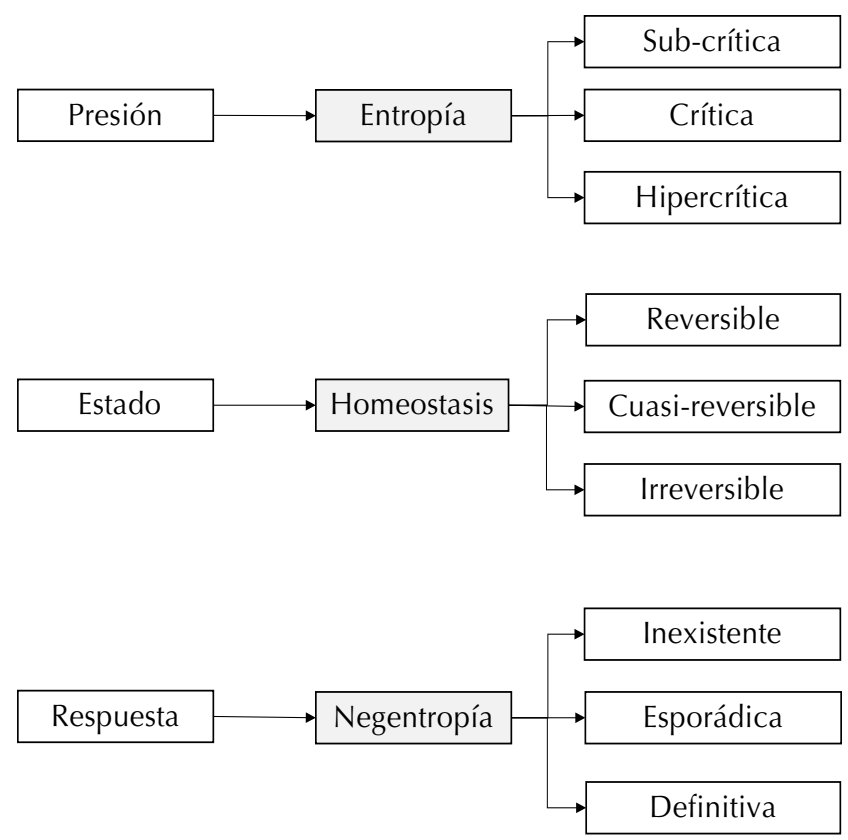

Figura 1. Estructura del modelo EHN

Fuente: González (2018).

\section{MÉTODO}

Se realizó un estudio cualicuantitativo (Hernández, Fernández y Baptista, 2003), considerando la información vertida por el Siteur. Concretamente, datos referidos a la línea 2, desde la estación Juárez hasta la estación Tetlán, tal y como se muestra en la figura 2. La justificación se basa en el hecho de que por esta línea se encuentran estaciones clave de afluencia poblacional indicadas en la figura 3. Estos puntos son estratégicos geográfica, comercial y/o turísticamente. La estación Tetlán, el mercado San Juan de Dios y el centro histórico de Guadalajara son algunos ejemplos de nodos de importancia urbana. En este sentido, durante las denominadas horas pico, desde las 18:30 horas hasta las 20:20 horas se observó la frecuencia en el paso de los trenes, la cantidad promedio contable de usuarios que caben dentro de un carro de una unidad del tren eléctrico (dos carros/unidad), así como los costos económicos y gastos energéticos que presenta el sistema ante la CFE. 


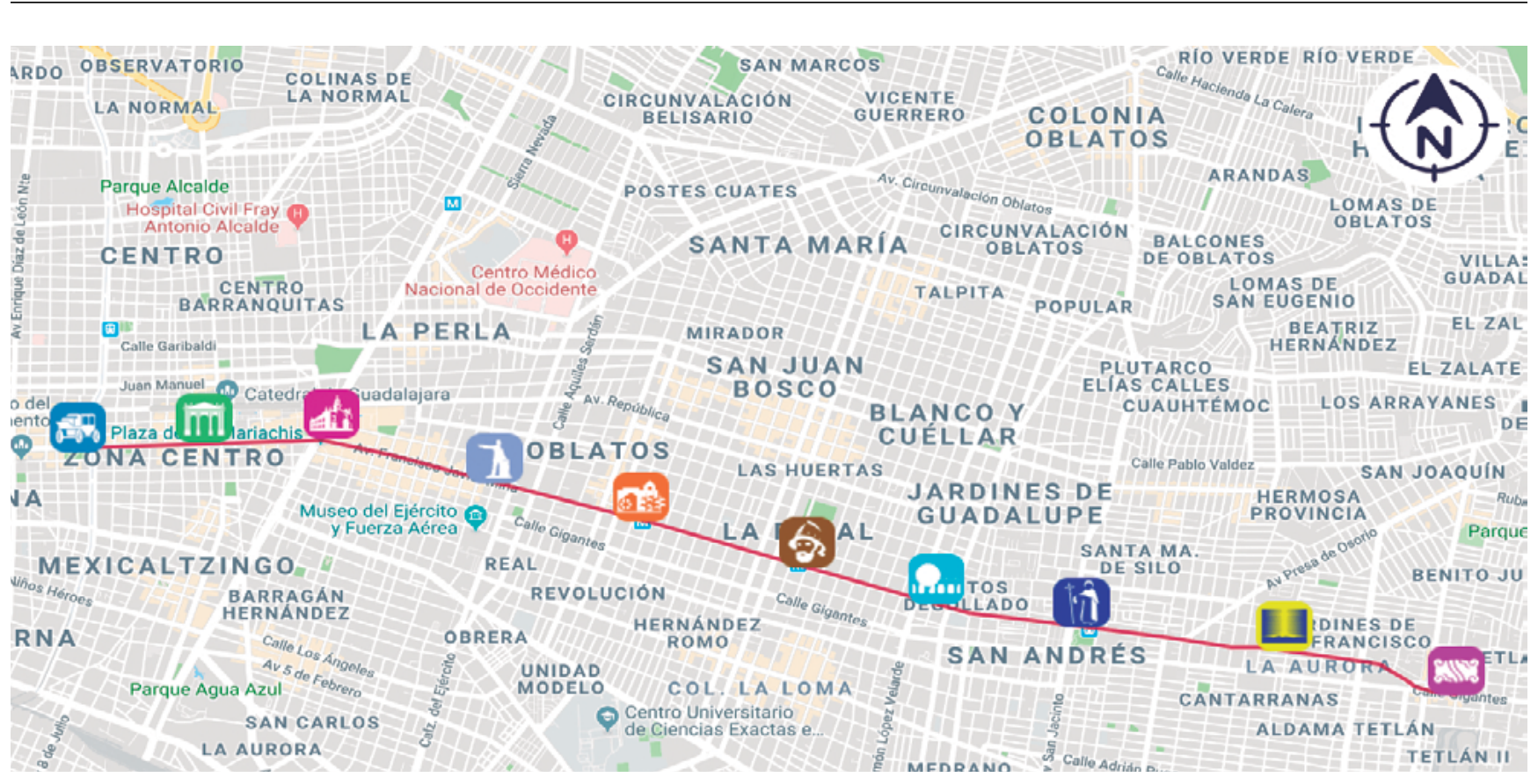

Figura 2. Línea 2 del Siteur (oriente-occidente)

Fuente: Siteur (2019).

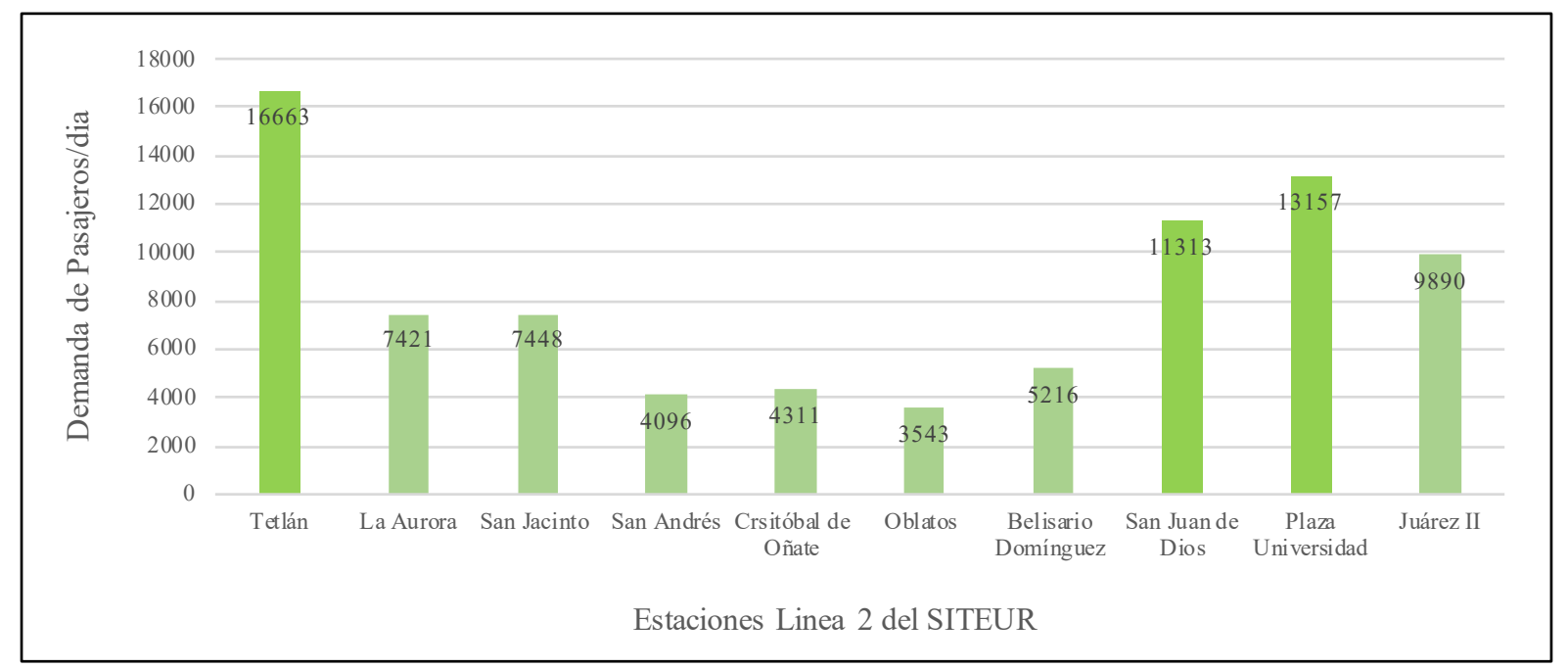

Figura 3. Afluencia en la línea 2 del Siteur

Fuente: ISA (2016).

Durante el tiempo que se estuvo observando la frecuencia de paso de los trenes en las horas pico, fue posible constatar que la línea 2 cuenta con dos modelos de trenes en operación y que han brindado el servicio por más de 20 años, los cuales para identificarlos se denominaron carro 1 al modelo
TEG-88 y carro 2 al modelo TEG-90. Asimismo, se revisaron datos proprocionados en 2017 por Siteur y la Comisión Federal de Electricidad (CFE). Se realizó, además, un estudio de campo a través de un conteo manual in situ para obtener una cantidad promedio de usuarios que entran a un carro del 
tren ligero durante las horas de mayor demanda; es decir, se consideró el peso promedio total de personas dentro de un carro del tren ligero, que abordaron la línea 2 en estos horarios, y desde la estación Juárez (occidente) a la estación Tetlán (oriente). Este conteo permitió comparar el consumo de energía eléctrica al momento de mover las unidades, con respecto al porcentaje máximo de peso de los usuarios.

Aunado a lo anterior, se utilizaron datos del consumo eléctrico durante 2017, donde se identificó la cantidad de energía diaria, semanal y mensual que consume el tren ligero en su tracción para su movimiento y su servicio. Asimismo, tomando en cuenta los precios de las tarifas de alta tensión de la CFE considerando la demanda energética por región, sobre la distribución eléctrica que alimenta a las líneas del tren ligero. Para ello, se identificó un promedio estándar de la cantidad de personas que hacen uso del sistema desde a las 6:30 horas hasta las 20:20 horas en la línea 2 del Siteur; bajo el supuesto teórico de que el tren viaja con una cantidad constante de carga de extremo a extremo; además, se considera la utilización hipotética de sistemas piezoeléctricos para comparar los gastos del Siteur ante la CFE.

La investigación centró su estudio en la línea 2 del tren eléctrico, donde se observó la frecuencia de paso de los trenes en las horas pico; desde las 18:40 horas hasta las 20:20 horas. En este sentido, se eligió como punto de partida la estación Juárez; una de las más importante del Siteur. Esta se ubica en la zona centro de la ciudad de Guadalajara y en horas pico cuenta con una gran cantidad de viajes desde las 16:30 hasta las 20:16 horas. Asimismo, se tuvieron 6 carros tipo TEG-90 y 4 carros tipo TEG-88, en donde fue considerada la cantidad de usuarios dentro de una unidad de tren ligero, mediante conteos realizados manualmente, para obtener una media total de en la unidad, tal y como se muestra en las figuras 4 y 5 .

Las observaciones y toma de mediciones mostradas en la tabla 1 fueron realizadas el día martes 5 de diciembre de 2017, con el objetivo de corroborar la frecuencia de paso de los trenes en las horas pico (18:40 y 20:20 horas). En este sentido, en esta última hora se observó una considerable disminución la cantidad de usuarios que pretendían abordar el tren dentro de la estación Juárez en dirección a la estación Tetlán.

El cálculo del voltaje real que se puede producir con un sistema piezoeléctrico consideró los estándares establecidos por Academia de Ciencias de Morelos en México (2010), a través de las ecuaciones (1) a (3).
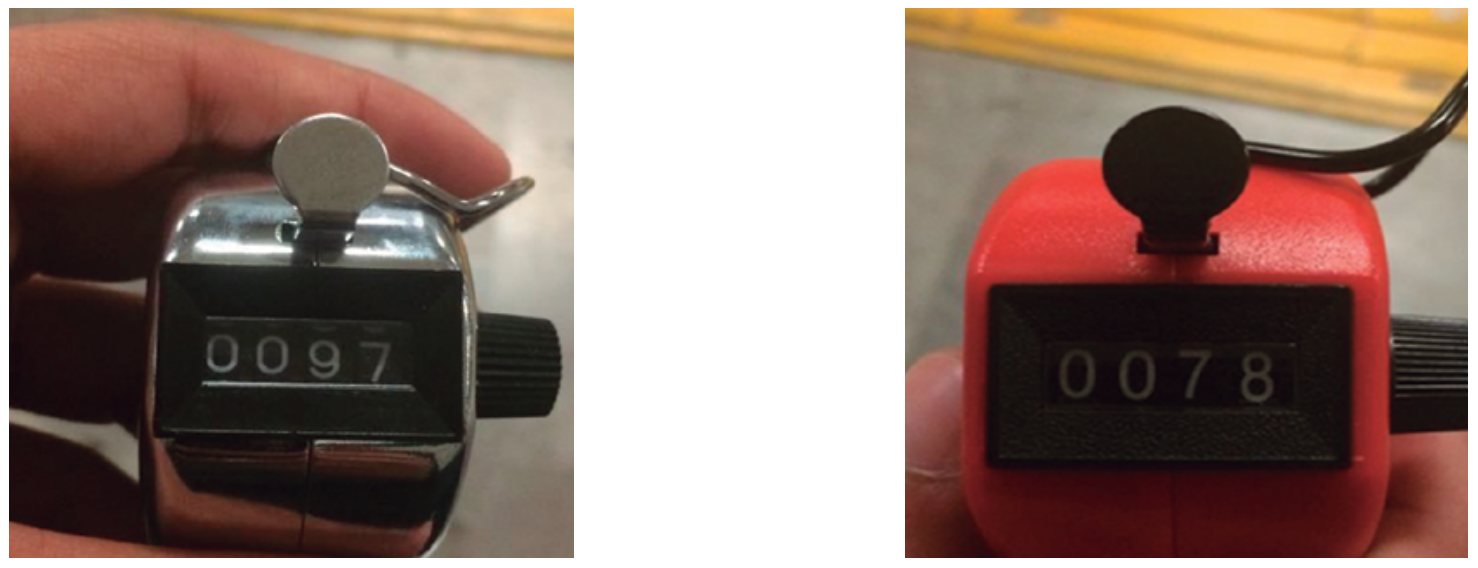

Figura 4. Contadores manuales utilizados en el ejercicio de campo

Fuente: elaboración propia. 


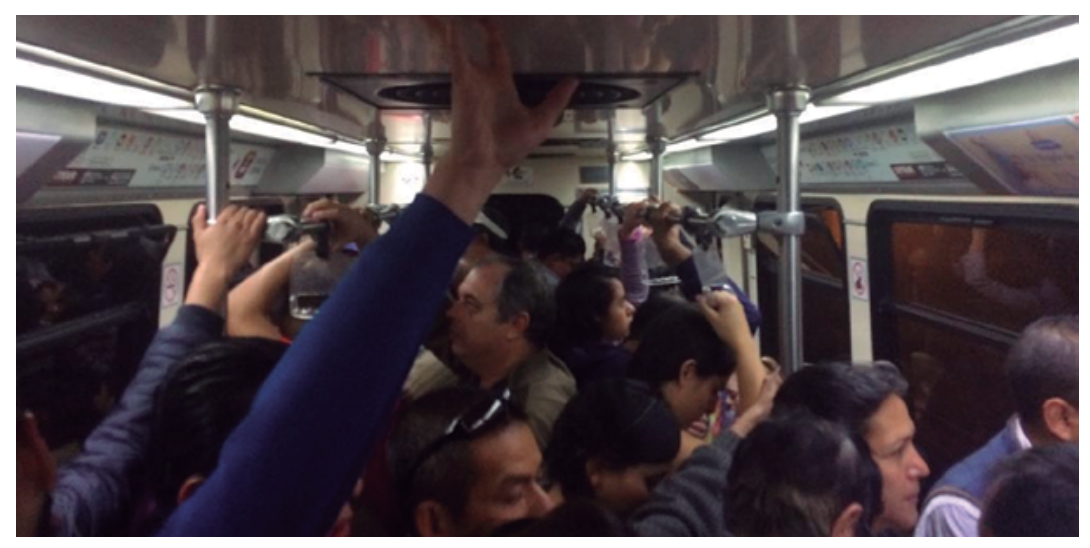

Figura 5. Usuarios de pie, en un carro de la línea 2 del tren eléctrico de Guadalajara

Fuente: elaboración propia.

Tabla 1. Observaciones in situ en la línea 2 del Siteur

\begin{tabular}{cl}
\hline Hora & \multicolumn{1}{c}{ Observaciones } \\
\hline 18:30 & $\begin{array}{l}\text { Hora de llegada a la estación Juárez en dirección a la estación Tetlán sobre la línea 2; haciendo presencia en } \\
\text { persona para observar el momento en el que se empieza a congestionar la estación de usuarios que pretenden } \\
\text { abordar el tren durante ese periodo de tiempo, y así definir el inicio de la hora pico dentro de la estación. }\end{array}$ \\
\hline $18: 44$ & $\begin{array}{l}\text { Empieza a aglomerarse los usuarios en el andén de espera para abordar al tren. } \\
\text { 18:46 }\end{array}$ \\
\hline $\begin{array}{l}\text { Inicia la hora pico, ya que se pudo observar un incremento de hasta un } 50 \% \text { adicional de usuarios que esperaban } \\
\text { al tren. Se logra identificar esta situación, debido al total de personas que estaban esperando en el andén. Asimis- } \\
\text { mo, solo el 50 \% logra su abordaje. }\end{array}$ \\
\hline $18: 50$ & $\begin{array}{l}\text { A partir de este momento, los carros de los trenes que estaban en servicio empiezan a desplazarse totalmente } \\
\text { Ilenos, de la estación Juárez con dirección a Tetlán en la línea 2. }\end{array}$ \\
\hline 19:30 & $\begin{array}{l}\text { En este periodo de tiempo, continúa llegando gran cantidad de usuarios que abordan y descienden dentro de la } \\
\text { estación Juárez. }\end{array}$ \\
\hline 20:10 & $\begin{array}{l}\text { Ocurre una disminución gradual de usuarios que esperan en el andén para abordar a los carros del tren ligero } \\
\text { hasta en un 50\%. }\end{array}$ \\
\hline $\begin{array}{l}\text { Concluye la hora pico en la estación Juárez con dirección a Tetlán en la línea 2. Durante el tiempo que se hizo } \\
\text { presencia, se pudo observar que Siteur mantiene un patrón de movilización en sus unidades dentro de la línea 2, } \\
\text { ya que hacían uso de sus dos modelos de trenes que se pueden identificar fácilmente, y los cuales intercambiaban } \\
\text { conforme a los viajes. }\end{array}$ \\
\hline
\end{tabular}

Fuente: elaboración propia.

Donde:

$$
\begin{gathered}
\mathrm{V}=\mathrm{g} 33 \mathrm{hT} \\
\mathrm{F}=\mathrm{mg} \\
P=\frac{\mathrm{F}}{\mathrm{A}}
\end{gathered}
$$

$\mathrm{V}=$ voltaje.

g33 = constante de voltaje del piezoeléctrico.

h = altura (grosor) del cerámico.

$$
\begin{aligned}
& T=\text { presión sobre el elemento. } \\
& \mathrm{F}=\text { fuerza. } \\
& \mathrm{m}=\text { masa. } \\
& \mathrm{g}=\text { aceleración de la gravedad. } \\
& \mathrm{P}=\text { presión. } \\
& \mathrm{F}=\text { fuerza. } \\
& \mathrm{A} \text { = área. }
\end{aligned}
$$




\section{RESULTADOS}

Se identificaron 6 trenes del modelo TEG-90 y 4 trenes del modelo TEG-88 operando en horas pico. De ahí, fueron contabilizados 27 viajes en un tiempo de 1,36 horas; considerando un tiempo de recorrido en 15 minutos y una distancia de 8,5 km. Asimismo, se obtuvo una cantidad aproximada de 235 usuarios a bordo. En este sentido, asumiendo un peso promedio del mexicano de $75 \mathrm{~kg}$; entonces, la carga transportada oscila en los $17.625 \mathrm{~kg}$ por recorrido. No obstante, una unidad consta de dos carros y, suponiendo que ambos carros cargaran la misma cifra de usuarios en condiciones similares de masa corporal, se tendrían 35,25 toneladas por recorrido. De esta manera, para el cálculo del voltaje real se utilizó la ecuación (1), donde primeramente se obtuvo la fuerza de presión con la ecuación (2) y posteriormente la presión ejercida con la ecuación (3), sobre el área de los cristales piezoeléctricos $\left(0,012 \mathrm{~m}^{2}\right)$, el resultado corresponde a $61.312,5 \mathrm{P}$

De acuerdo con el grosor del cerámico utilizado por la Academia de Ciencias de Morelos (ACMor), para sus discos piezoeléctricos, el valor correspondiente para este estudio es $h=0,14 \mathrm{~mm}$, o bien, $\mathrm{h}=0,00014 \mathrm{~m}$. Por tanto, el voltaje real que se obtendría por el material piezoeléctrico de acuerdo con la ecuación (1) es de 17,168 V.

El voltaje anterior sería el total que se produciría con la constante de voltaje piezoeléctrica de los discos para una persona con un peso de $75 \mathrm{~kg}$.
En este contexto, tomando la cantidad de 235 pasajeros contabilizados durante un viaje en las horas pico de las 18:30 y 20:20 horas se tiene dentro de un carro; es decir, media unidad de tren ligero un $\mathrm{Vr}=17,1675 \mathrm{~V}$. Así pues, para 235 usuarios de $75 \mathrm{~kg}$ cada uno, se obtiene 4034,3 V $(4,03 \mathrm{kV})$.

Por otro lado, en dos carros o una unidad completa del tren ligero, considerando que lleva la misma cantidad de usuarios y los mismos sistemas piezoeléctricos se tiene que para 470 usuarios del mismo peso se obtienen 8068,7 V (8,06 kW). Esta cantidad es suficiente para alimentar las tensiones reglamentarias, que oscilan entre los $0,75 \mathrm{~kW}, 1,0$ kW y 3,0 kW. En este sentido, "una persona de 68 $\mathrm{kg}$ con un porcentaje de grasa corporal del $15 \%$ equivale a una energía química acumulada de 348 MJ" (Starner y Paradiso, 2004). De tal forma que si esta persona realiza una frecuencia de 2 pasos/ segundo puede llegar a generar un promedio de 67 W (Ibáñez, 2012); esto implica entonces, que 470 usuarios con un peso de $68 \mathrm{~kg}$ cada uno generarían 31.490,0 W (31,49 kW). Por tanto, considerando los 27 viajes contabilizados durante las horas pico vespertinas, con 470 usuarios del mismo peso, se tendría entonces, una capacidad de generación de energía de 850,23 kWh. Esta cantidad se encuentra relativamente cerca de la energía consumida en 2017 por el sistema (947,41 kWh). En suma, es posible lograr un ahorro considerable el cual se muestra en la figura 6 .

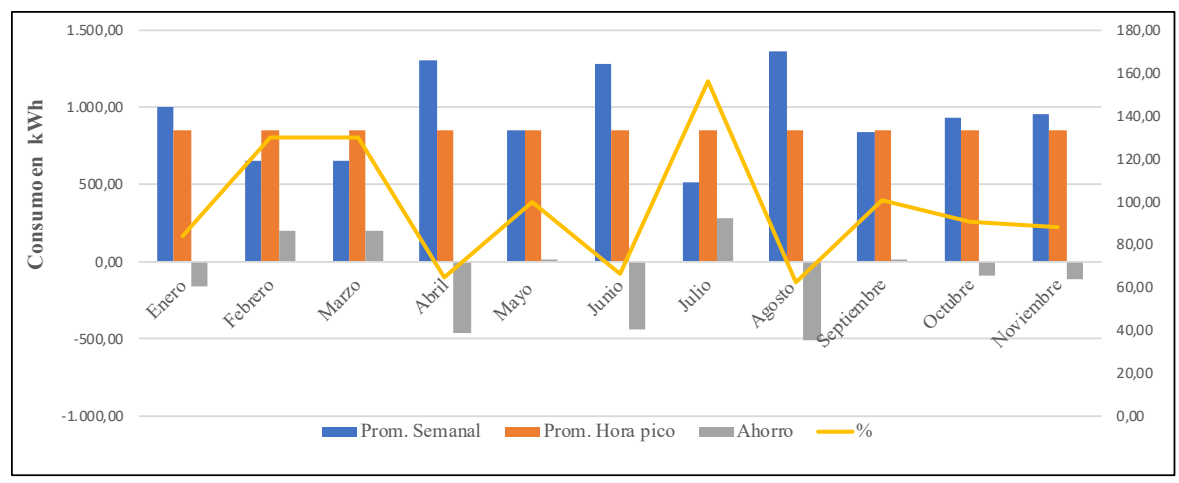

Figura 6. Ahorro promedio mensual en kWh Generación de energía en hora pico de enero-noviembre de 2017.

Fuente: elaboración propia. 
Es preciso mencionar que los datos anteriormente mencionados son conservadores respecto al supuesto de que solo una unidad del servicio de tren ligero haga su recorrido con los datos límite de usuarios y su peso.

\section{CONCLUSIÓN}

Las energías renovables están tomando el papel principal de generación de energía en todo el mundo, ya que se considera de gran necesidad mundial el ser más eficientes y sustentables como sociedad.

Actualmente, grandes empresas han optado por implementar fuentes renovables de energía, con la finalidad de contrarrestar el impacto ambiental producido por la forma de habitar y moverse en la ciudad. En este contexto, el Siteur ha demostrado las ventajas de la innovación tecnológica y el uso de fuentes de energía no dependientes de los recursos fósiles. Recientemente, ha sustituido los rehiletes en cada una de sus estaciones, por unos automatizados donde se utiliza una tarjeta de prepago (InnovaCard) para poder acceder al ingreso de las estaciones y utilizar el servicio. Este nuevo mecanismo se implementó a partir de abril de 2018, con la finalidad de crear y facilitar la movilidad de los usuarios. Sin embargo, la gran afluencia que tienen las estaciones durante las horas pico origina entropía o energía no utilizada para producir trabajo. De esta manera, en estos horarios, es posible aprovechar la aglomeración de usuarios para generar energía que permita alimentar al sistema, el cual se indica en la tabla 2.

Ciertamente, el uso de la tecnología piezoeléctrica en áreas de movilidad viene cobrando gran importancia e interés en el mundo, ya que faculta la generación de energía a través de sistemas que favorecen la mitigación del impacto ambiental ocasionado por el alto consumo energético. Esta tecnología tiene una gran versatilidad en su implementación, toda vez, que puede ser instalada dentro de cada unidad del tren, en pasillos o escaleras y aprovechar el peso de cada usuario.

Un sistema piezoeléctrico en condiciones óptimas de generación puede generar una eficiencia de hasta el 89,7 \% en comparación con el promedio semanal de consumo de kW del Siteur sobre la línea 2; esto debido a la gran afluencia que en promedio ronda los 83.059 usuarios diarios en horas pico. Por tanto, es posible generar energía eléctrica a través de estos sistemas y la entropía originada por la conglomeración y afluencia de usuarios en el Siteur; posteriormente, esta energía puede ser almacenada o inyectada en las líneas de la red para la tracción y funcionamiento del tren. Este escenario implicaría considerables ahorros económicos, eficiencia y beneficios diversos para la empresa (Siteur), convirtiéndose en un sistema de transporte sustentable, al generar y operar con fuentes alternas de energía.

Tabla 2. Aplicación del modelo EHN a la línea 2 del Siteur

\begin{tabular}{lll}
\hline \multicolumn{1}{c}{ Entropía } & Homeostasis & \multicolumn{1}{c}{ Negentropía } \\
\hline $\begin{array}{l}\text { Conglomeración y afluencia de usuarios } \\
\text { dentro de los carros del tren ligero y es- } \\
\text { taciones del Siteur durante la hora pico. }\end{array}$ & $\begin{array}{l}\text { Uso de recursos renovables para la gene- } \\
\text { ración de energía. }\end{array}$ & $\begin{array}{l}\text { Gasto en el consumo de energía, debido a } \\
\text { la alta demanda del servicio en especial en } \\
\text { horas pico y días festivos. }\end{array}$ \\
\hline $\begin{array}{l}\text { Secuencia de paso de los carros del tren } \\
\text { ligero durante las horas pico. }\end{array}$ & $\begin{array}{l}\text { Aprovechamiento de la afluencia y con- } \\
\text { glomeración con el peso de los usuarios } \\
\text { para la generación de energía con tecno- } \\
\text { logía piezoeléctrica. }\end{array}$ & $\begin{array}{l}\text { Pago de impuestos por parte del Siteur, por } \\
\text { el uso la energía para brindar el servicio, } \\
\text { la CFE para el subministro de energía. }\end{array}$ \\
\hline $\begin{array}{l}\text { Gasto y consumo energético por los } \\
\text { servicios del Siteur. }\end{array}$ & $\begin{array}{l}\text { Interacción de la tecnología piezoeléctri- } \\
\text { ca para la generación de energía con la }\end{array}$ & $\begin{array}{l}\text { Gasto y consumo energético por los servi- } \\
\text { cios de Siteur. }\end{array}$ \\
\hline
\end{tabular}

Fuente: elaboración propia. 


\section{REFERENCIAS}

Academia de Ciencias de Morelos (ACMor) (2010). Pisando y generando piezoelectricidad. Recuperado de: http://acmor.org.mx/cuamweb/reportescongreso/2010/fisico-mate/110-\%20CUM-\%20Pisando\%20y\%20Generando.pdf

Comisión Federal de Electricidad (CFE) (2017). Esquemas Tarifarios. Recuperado de: https://app.cfe.mx/Aplicaciones/CCFE/Tarifas/Tarifas/Tarifas_negocio.asp

Faure, R. (2004). La tracción eléctrica en la alta velocidad ferroviaria (A.V.F.). Madrid: Colegio de Ingenieros de Caminos, Canales y Puertos. doi: https:// doi.org/10.3989/ic.1969.v22.i210.3718

García, F., González, M. y Asprilla, Y. (2018). Determinación de los componentes entrópicos de la accidentalidad: el trinomio vehículo/usuario/ camino en la metrópoli de Guadalajara, México. Tecnura, 22(55), 51-65. doi: https://doi.org/ 10.14483/22487638.13245

González, M. (2016). Infraestructura y desarrollo: las afectaciones del comercio adyacente a la línea 3 del tren ligero en Jalisco. Tecnogestión, 1(13), 2434. doi: https://doi.org/10.5821/ace.12.36.4848

González, M. (2018). Entropy and negentropy of the particular electric vehicle in urban systems: homeostasis of mobility in Mexico. DYNA, 85(206), 171-177. doi: https://doi.org/10.15446/dyna. v85n206.72509

De Quevedo, F., Asprilla, Y. y González, M. (2017). Entropías de la movilidad urbana en el espacio metropolitano de Guadalajara: transporte privado y calidad del aire. Tecnura, 21(53), 138-149. doi: https://doi.org/10.14483/22487638.10725

Hernández, R., Fernández C. y Baptista, P. (2003). Metodología de la investigación. México: McGraw-HiII Interamericana.

Ibáñez, J. (2012). Diseño de un prototipo para la generación energética mediante tecnología piezoeléctrica. Aplicación a escaleras. Recuperado de https://upcommons.upc.edu/bitstream/ handle/2099/14982/lb\%C3\%A1\%C3\%B1ez. pdf? sequence $=1$ \&is Allowed $=y$

Instituto de Información Estadística y Geografía del Estado de Jalisco (IIEG) (2017). Estudio de población y sociedad. Información sociodemográfica urbana. Jalisco. doi: https://doi.org/10.2307/j.ctvg5bs7s.9

ISA Corporativo (2016). Presentación de datos estadísticos de afluencia en SITEUR. Recuperado de http:// isa.com.mx/pdf/PRESENTACION_SITEUR.pdf.

Mapa Metro (2010). Tren Eléctrico de Guadalajara. doi: https://doi.org/10.14482/sun.30.3.7101

Sistema de Transporte Colectivo (2007). Operación. Cifras operacionales. Recuperado de: http://www.metro.df.gob.mx.

Siteur (2014). Innovación y Tecnología de los Trenes. doi: https://doi.org/10.3989/revmetalm.2005.v41. i2.194

Siteur (2019). Línea 2. Recuperado de http://www.siteur. gob.mx/tren-ligero/linea-2.html

Starner, T. y Paradiso, J.A. (2004). Human Generated Power for Mobile Electronics. En C. Piguet (ed.), LowPower Electronics Design (pp. 1-35). CRC Press. doi: https://doi.org/10.1201/9781420039559.ch45

\section{(c) (1) () ()}

